\title{
Synthesis and Characterization of Glassy Carbon Nanowires
}

\author{
C. M. Lentz, ${ }^{1}$ B. A. Samuel, ${ }^{2}$ H. C. Foley, ${ }^{3}$ and M. A. Haque ${ }^{2}$ \\ ${ }^{1}$ Department of Materials Science and Engineering, The Pennsylvania State University, University Park, PA 16802, USA \\ ${ }^{2}$ Department of Mechanical and Nuclear Engineering, The Pennsylvania State University, University Park, PA 16802, USA \\ ${ }^{3}$ Department of Chemical Engineering, The Pennsylvania State University, University Park, PA 16802, USA
}

Correspondence should be addressed to M. A. Haque, mah37@psu.edu

Received 7 June 2010; Accepted 13 September 2010

Academic Editor: Teng Li

Copyright (๑) 2011 C. M. Lentz et al. This is an open access article distributed under the Creative Commons Attribution License, which permits unrestricted use, distribution, and reproduction in any medium, provided the original work is properly cited.

The advent of carbon-based micro- and nanoelectromechanical systems has revived the interest in glassy carbon, whose properties are relatively unknown at lower dimensions. In this paper, electrical conductivity of individual glassy carbon nanowires was measured as a function of microstructure (controlled by heat treatment temperature) and ambient temperature. The semiconducting nanowires with average diameter of $150 \mathrm{~nm}$ were synthesized from polyfurfuryl alcohol precursors and characterized using transmission electron and Raman microscopy. DC electrical measurements made at $90 \mathrm{~K}$ to $450 \mathrm{~K}$ show very strong dependence of temperature, following mixed modes of activation energy and hopping-based conduction.

\section{Introduction}

Semiconducting amorphous materials are widely used in electronics applications due to their favorable optical and dielectric properties, tunable band gaps, and low manufacturing costs [1-3]. Amorphous or glassy carbon $(\mathrm{a}-\mathrm{C})$ is of particular interest because of the versatility of the carbon material system with a diverse range of physical properties based on the nature and the spatial arrangement of the chemical bonds [4]. A common method of glassy carbon synthesis is the pyrolysis of a polymeric precursor. Such polymer-derived carbons are considered to be either graphitizing or nongraphitizing depending on their tendency to transform to graphite when subjected to temperatures above $1000^{\circ} \mathrm{C}$. Nongraphitizing carbons are globally amorphous, meaning that they are disordered and noncrystalline though they may have some small graphitic regions with local order. Though a long range graphitic structure is the most thermodynamically stable form of carbon, nongraphitizing carbons remain thermally stable and resistant to chemical attack at high temperature. This stability is attributed to crosslinking in the polymeric precursor, which results in a disordered, chaotic misalignment of graphene layers in the carbon after pyrolysis [5].

The composition of the carbon-carbon bond types in a given glassy carbon material is, along with the material microstructure, one of the most influential parameters in determining the electrical transport properties. The presence of more delocalized $\pi$ electrons due to $\mathrm{sp}^{2}$ bonding facilitates in-plane electron transport in graphitic carbon, which is not the case for the rigid and localized $\mathrm{sp}^{3}$ bonds in diamondlike carbons. In tetrahedral carbon systems, for instance, an increase in electrical conductivity is generally observed with increase in the $\mathrm{sp}^{2} / \mathrm{sp}^{3}$ ratio in the material [6]. Therefore, even in materials with a low $\mathrm{sp}^{2} / \mathrm{sp}^{3}$ ratio, it is the $\mathrm{sp}^{2}$ arrangement that determines the electrical properties of the material. A range of band gaps from $1-4 \mathrm{eV}$ is seen in the literature for glassy carbon [7-9], where variations arise from the composition of $\mathrm{sp}^{3}$ and $\mathrm{sp}^{2}$ carbon and from defect induced variation in the density of states.

In this study, we investigate the role of microstructure and ambient temperature on the electrical properties of glassy carbon, which has a disordered structure with primarily $\mathrm{sp}^{2}$ carbon. Though lacking in long-range order, these carbons are regular solids; they contain structural elements of the same type that are chaotically arranged in space. This regularity is observed in their inherent nanoscale porosity, which is narrowly distributed around an average pore size of $0.5 \mathrm{~nm}$ [10]. Such nanoporous carbons have found applications in filtration, electrical [11-13], and electrochemical applications [14-16]. The motivation for this study comes from a resurgence of the interest in nano 
[14, 17-20] and biological [21, 22] applications of glassy carbon materials. Carbon is clearly a more suitable material over silicon for biological applications because life on earth is carbon-, and not silicon-based. In particular, a new area called "carbon MEMS (micro-electro-mechanical systems) is developing rapidly. The uniqueness of the technique is that nanofabrication processes are cleverly exploited to pyrolize polymeric nanostructures to carbon-MEMS that can range from micro- to nanoscale [23] and different aspect ratios [24]. It is important to note that all these applications require comprehensive characterization of the electrical conductivity of the nanoscale glassy carbon. The literature contains ample information on the bulk glassy carbon properties [7-9, 25, 26], but only a few studies are available that investigate the size effect on electrical properties at the nanoscale [27, 28]. For example, compared to microporous bulk glassy carbon, a nanoporous nanowire has higher surface area/volume ratio, as well as defects due to the presence of 5- and 7-membered aromatic rings and uncoordinated carbon surface atoms. Because of these defects, many of the charge carriers in the system exist in localized states in the semiconductor band gap, altering the electrical transport properties of the material.

\section{Literature Review}

2.1. Synthesis and Structure. Glassy carbon is typically synthesized by pyrolizing any polymeric precursor that crosslinks at elevated temperatures. Specifically, the nanowires synthesized in this study are based on polymerized furfuryl alcohol (PPFA), which is a common furanic derivative of natural biomasses [29]. Fitzer and Schäfer [30] used infrared spectroscopy (IR) to examine the mechanism for the pyrolysis of PFA, at increasing temperatures from a starting point of $130^{\circ} \mathrm{C}$. The furan ring remains stable up to $275^{\circ} \mathrm{C}$, only fully rupturing between 300 and $400^{\circ} \mathrm{C}$. At $400^{\circ} \mathrm{C}$, aromatic groups begin to form spontaneously from the fragments of the furan rings. As temperature increases above $450^{\circ} \mathrm{C}$, the aromatic groups coalesce to form a final, highly unsaturated polymer network. Between 500 and $1200^{\circ} \mathrm{C}$, called the dehydrogenation phase, hydrogen atoms are expelled, and the linear conjugated links coalesce to form aromatic systems. The final phase is annealing, which occurs at heat treatment above $1200^{\circ} \mathrm{C}$. During this stage, structural defects such as five- and seven-membered rings are progressively removed from the system as temperature is increased. Multiple models for the structures of nongraphitizing carbons exist. The final microstructure is composed of randomly oriented graphitic crystallites connected by disordered carbon sheets, or a three-dimensional network of curved graphitic ribbons, which loop around each other in no specific orientation [31-33].

2.2. Electrical Characterization of Glassy Carbon. Glassy carbons are considered to be Anderson insulators, where the systemic disorder, through particle localization, induces a transition from metallic conduction to an increasingly insulating state as disorder increases [34]. This is a strong function of structural order, which is driven by stress relaxation [35]. For heat treatment temperatures above $500^{\circ} \mathrm{C}$, rapid removal of hydrogen atoms produce mobile carriers via a method in which an edge carbon atom attracts electrons from a carbon atom in the interior of the aromatic sheet, producing a negatively charged edge ion and a mobile interior hole. This results in an increase in the number of mobile carriers, causing the steep decrease in resistivity. However, this process slows down exponentially after $1000^{\circ} \mathrm{C}$.

In disordered carbon the charge carrier transport occurs in three regimes-tunneling transport between the localized states in the mobility gap at very low temperatures $(<20 \mathrm{~K})$, hopping-based conduction between localized states at low temperatures $(<250 \mathrm{~K})$ and thermally-activated conduction from the Fermi level to the mobility edges at room temperature and higher [36]. The hopping-based charge transfer occurs by phononassisted tunneling from occupied to unoccupied electronic states. The hopping conduction model predicts a $T^{-1 /(d+1)}$ relationship with the logarithm of the conductivity, where $d$ is the dimensionality of the hopping space [33],

$$
\sigma(T)=\sigma_{0} \exp \left(-\left[\frac{T_{0}}{T}\right]^{1 /(d+1)}\right) .
$$

For glassy carbon, hopping is commonly observed to be three dimensional $(d=3)$ in a range of ambient temperatures from $60 \mathrm{~K}$ to $300 \mathrm{~K}$ [37]. With hopping conduction, the conductivity approaches zero as temperature approaches zero. In addition to the effects of localized states, a Coulomb gap can have an effect on the hopping conductivity mechanism [33], resulting in a $T^{-1 / 2}$ dependence of logarithm of conductivity, regardless of hopping space dimension. This is given by,

$$
\sigma=\sigma_{0} \exp \left\lfloor-\left(\frac{T_{0}}{T}\right)^{1 / 2}\right\rfloor, \quad \text { where } T_{0}=\frac{6 e^{2}}{\pi k_{B}} \frac{1}{4 \pi \varepsilon} \frac{1}{\xi} .
$$

Here, $\sigma_{0}$ is the conductivity at $T=\infty, \varepsilon$ is the permittivity representing screening effects on charges at small graphitic regions, and $\xi$ is the localization length of the wave function, defined as the spatial extension of the localized energy state. The Coulomb gap is calculated to be equal to $k_{B} e^{4} D_{0} \xi / \varepsilon$, where $D_{0}$ is the density of states at the Fermi energy. The contribution of the Coulomb interactions can occur when the thermal energy is smaller than the Coulomb gap width. This occurs below a characteristic temperature, $T_{C}=$ $\left(e^{4} D_{0} \xi\right) / \varepsilon^{2}$.

At or above room temperature, thermally-activated transport mechanism is expected. Here, the relationship between $\ln$ (conductivity) and surface temperature is indicative of the activation energy gap between the Fermi level and the extended states in the disordered carbon's mobility edge, $\left(\varepsilon_{g}=\varepsilon_{V}-\varepsilon_{F}\right)$,

$$
\sigma(T)=\sigma_{0} e^{-\varepsilon_{g} / k_{B} T},
$$

where $\sigma_{0}$ is a constant, and $k_{B}$ is the Boltzmann constant [22].

Bulk electrical properties of glassy carbon are very well studied in the literature. Kuriyama and Dresselhaus [34] 
studied the metal-insulator transition in activated phenolderived, nongraphitizing carbon fibers. They determine that the metal-insulator transition occurs at approximately $1000^{\circ} \mathrm{C}$, at which point the $\sigma \rightarrow 0$ as $T \rightarrow 0$ relationship begins to fail as fewer states are localized. Takai et al. [33] performed a study on a primarily $\mathrm{sp}^{2}$, nongraphitic carbon, heat-treated over a range of $200^{\circ} \mathrm{C}$ to $1500^{\circ} \mathrm{C}$. They observed variation in conductivity with both heat treatment temperature (HTT) and with ambient temperature over a range from $4.2 \mathrm{~K}$ to room temperature. With successive heat treatment at intervals of $\sim 200^{\circ} \mathrm{C}$ up to a $\mathrm{HTT} \leq 600^{\circ} \mathrm{C}$, the conductivity shows a drastic increase of four orders of magnitude. Wang et al. [31] observed the hopping conduction mechanism at temperatures from $75 \mathrm{~K}$ to $275 \mathrm{~K}$.

In comparison, very little is known about the lower dimensional properties of glassy carbon, which may be due to the fact that all the applications hitherto have been macroscopic. However, the advent of carbon-based microand nanodevices necessitates the fundament understanding in conduction at such reduced dimensions. Park et al. [38] studied the electrical properties of glassy carbon films derived from epoxy and phenolic resins and found the properties similar to bulk glassy carbon. Our previous study involved individual glassy carbon nanowires above room temperature to show about less than 1 order of magnitude change in conductivity as surface temperature increased from $300 \mathrm{~K}$ to $460 \mathrm{~K}$. All the specimens were heat treated at $600^{\circ} \mathrm{C}$ only, therefore the study did not show the role specimen microstructure and low temperature conduction [39].

\section{Experimental Techniques}

Furfuryl alcohol was polymerized inside the pores of an anodized alumina template to form the nanowires, which were subsequently pyrolized at temperature ranging from 600 to $2000^{\circ} \mathrm{C}$. In a typical process [10] $1 \mathrm{M} \mathrm{p}$ toluenesulfonic acid solution is added to $5 \mathrm{ml}$ of Triton X100. $5 \mathrm{ml}$ furfuryl alcohol (FA) is added to the resultant solution using a syringe pump at a rate of $10 \mathrm{ml} \mathrm{min}^{-1}$. The solution is allowed to polymerize (PFA) in an ice bath for $24 \mathrm{~h}$ and then filled inside Anodisc anodic alumina membrane (Whatman) with $200 \mathrm{~nm}$ pores by capillary effect. The PFA-lled template is then pyrolyzed for $8 \mathrm{~h}$ and then dissolved in $6 \mathrm{M} \mathrm{KOH}$ aqueous solution to produced dispersed nanowires. High temperature treatment (HTT) was carried out in a Red Devil furnace (R.D. Webb Company, Natick, MA). The hot zone was evacuated to $10^{-3}$ mbar for $24 \mathrm{~h}$ prior to annealing, then backfilled to atmospheric pressure with argon. A $25^{\circ} \mathrm{C} \mathrm{min}{ }^{-1}$ heating rate was employed with a $1 \mathrm{~h}$ soak at temperatures between 1200 and $2000^{\circ} \mathrm{C}$. Figure 1 shows the nanowire synthesis technique. The nanowires are then inspected with a JEOL $2010 \mathrm{LaB} 6$ transmission electron microscope to obtain information about the crystallite structure within the samples. The average diameter was found to be $150 \mathrm{~nm}( \pm 10 \mathrm{~nm})$, which is due to the volume shrinkage during pyrolysis.

Figure 2 shows the bright field and electron diffraction images for heat treatment temperatures of 600 and $2000^{\circ} \mathrm{C}$. The $600^{\circ} \mathrm{C}$ treated specimens (PPFA600) show no observable short-range structure, and the diffraction pattern shows Laue rings typical of glassy carbon, primarily diffused with two expected rings corresponding to the first and second coordination shells of carbon [39]. At higher temperatures $\left(800^{\circ} \mathrm{C}, 1200^{\circ} \mathrm{C}, 1500^{\circ} \mathrm{C}\right)$, graphitic clusters less than $10 \mathrm{~nm}$ in size appear, leading to ribbon-like morphology. The width of the Laue rings of the diffraction pattern appears narrower for these samples than for the lower temperature specimens. At $2000^{\circ} \mathrm{C}$ (PPFA 2000), we observe large ribbon-like chains $(<20 \mathrm{~nm})$ which are distributed in random orientation throughout the sample. The surface morphology of these nanowires also is jagged and different from all the other specimens. Therefore, from TEM microscopy we can conclude that the nanowires, while still remaining disordered, exhibit a remarkable decrease in the degree of disorder with increase in heat treatment temperature.

The TEM evidence of enhanced graphitic nanostructures at higher heat treatment temperatures is also corroborated by reflected light Raman spectroscopy performed on a clump of nanowires. The spectra were obtained on a WITec Confocal Raman instrument at an excitation wavelength of $514 \mathrm{~nm}$ and at 100X magnification. The results are shown in Figure 3. Peak broadening is observed at lower pyrolysis temperatures, which is indicative of bond length scatter (and disorder) at the molecular level in the form of strained bonds. With increasing heat treatment temperature some of these strains are relieved, possibly through contraction, expansion, or coalescence of $\mathrm{C}-\mathrm{C}$ bonds at the atomic scale. As shown in Figure 3, a sharp increase in the intensity of $G$ peak (compared to the $\mathrm{D}$ peak) is seen at $2000^{\circ} \mathrm{C}$, which implies that a sizeable number of the linear $\mathrm{sp}^{2}$ carbon have coalesced to form aromatic structures [40]. This is confirmed in the TEM image as an increase in the graphitic ribbon structures in PPFA2000. Therefore, Raman spectroscopy and TEM microscopy are complementary in deducing the evolution of the morphology of the nanowires.

Because the glassy carbon nanowires exhibited photosensitive electrical conduction, dark DC measurements with varying surface temperature of the nanowire (from $90 \mathrm{~K}$ to about $400 \mathrm{~K}$ ) were performed. Single nanowires were manipulated and bonded on microfabricated electrodes using focused-ion beam-based platinum deposition in a dual gun electron microscope (Quanta 200 3D Dual Beam FIB/SEM, FEI Company). This reduces the Schottky barrier and facilitate Ohmic conduction, as well as acting as a mechanical "glue" which both ensures that the nanowires are in contact with the surface and provides mechanical stability for the system. Measurements were carried out in a Deep Level Transient Spectroscopy (DLTS) cryogenic electronic test station, under vacuum. Conductivity measurements were made using a Keithley 236 Source-Measure Unit, sweeping voltage from $-1 \mathrm{~V}$ to $+1 \mathrm{~V}$ and measuring the resultant current. Figure 4 shows a typical specimen and electrical conductivity plot. Figure 4(b) shows a clear transition from one transport mechanism to another. However, the smooth transition of the curve indicates that multiple mechanisms work simultaneously in the transition regions. 


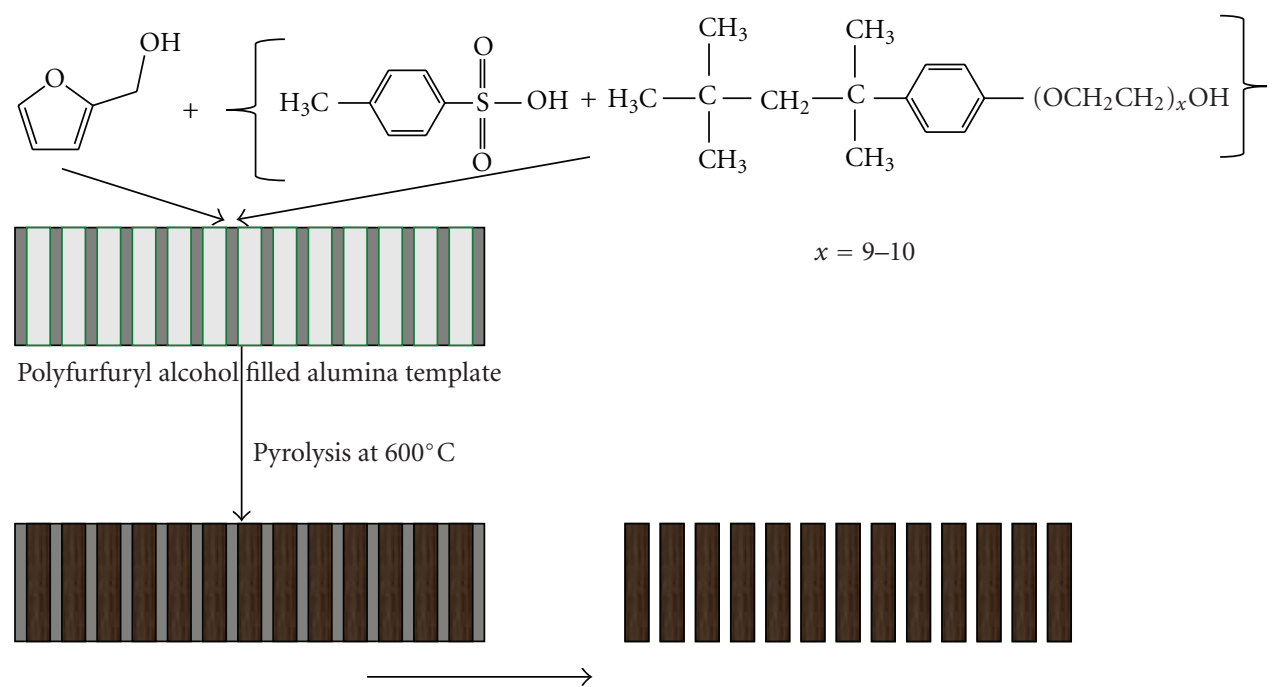

$\mathrm{KOH}$ Etch and heat treatment up to $2000^{\circ} \mathrm{C}$

FIGURE 1: Anodized alumina template-based synthesis of poly-furfuryl alcohol nanowires. The glassy carbon nanowires are obtained by pyrolyzing the polymeric precursor nanowires and then heat treating them at desired temperatures.

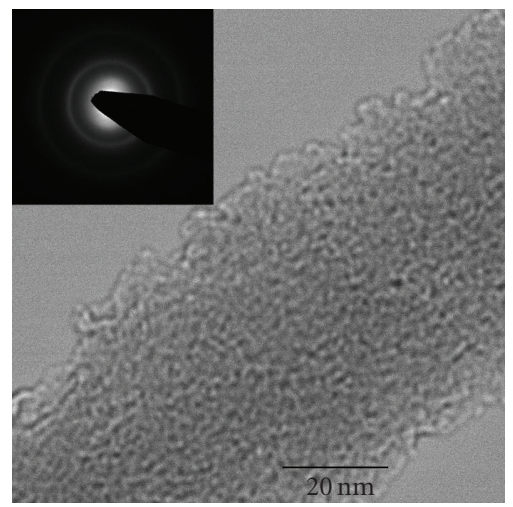

(a)

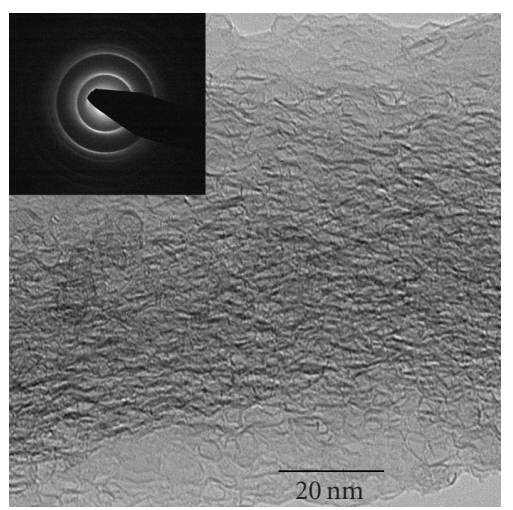

(b)

FIGURE 2: TEM bright field and diffraction patterns for glassy carbon nanowires treated at (a) $600^{\circ} \mathrm{C}$ and (b) $2000^{\circ} \mathrm{C}$ showing evolution of graphitic nanostructures.

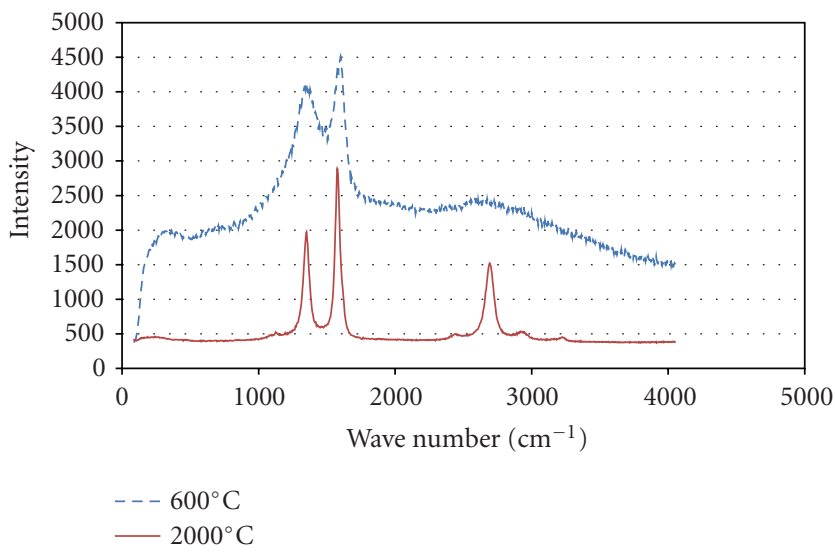

Figure 3: Raman spectra for nanowires heat treated at $600^{\circ} \mathrm{C}$ and $2000^{\circ} \mathrm{C}$. The peak sharpening at $2000^{\circ} \mathrm{C}$ agrees very well with the TEM analysis.

\section{Experimental Results and Discussion}

Electrical conductivity of individual glassy carbon nanowires were measured as a function of morphology and ambient temperature. The experimental results are plotted in Figure 5. As predicted by the TEM and Raman spectroscopy, the conductivity of the individual nanowires is observed to be strongly dependent on the specimen nanostructure. The increase in conductivity of the nanowires with increasing heat treatment temperature is due to the increases in aromatic $\mathrm{sp}^{2}$ ring formation, growth of curved graphitic crystallite domains and percolation networks, and the annealing of graphitic defects. Such increase in conductivity of the material by increasing the available density of charge transfer sites (and not by narrowing of the mobility gap) is analogous to an increase in doping in an extrinsic crystalline semiconductor. 


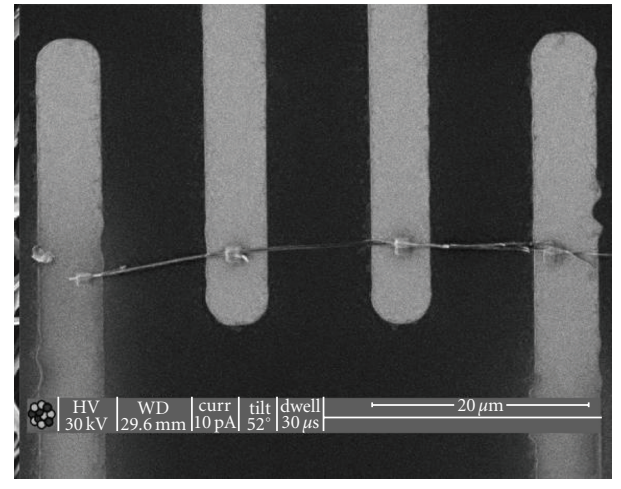

(a)

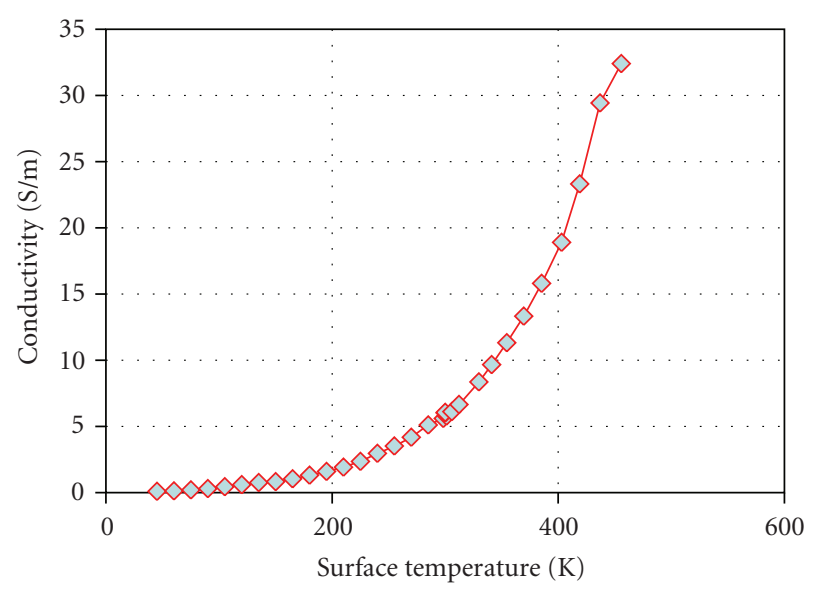

(b)

Figure 4: (a) Scanning electron micrograph of a single nanowire manipulated across interdigitated microelectrodes. (b) DC electrical conductivity of a nanowire.

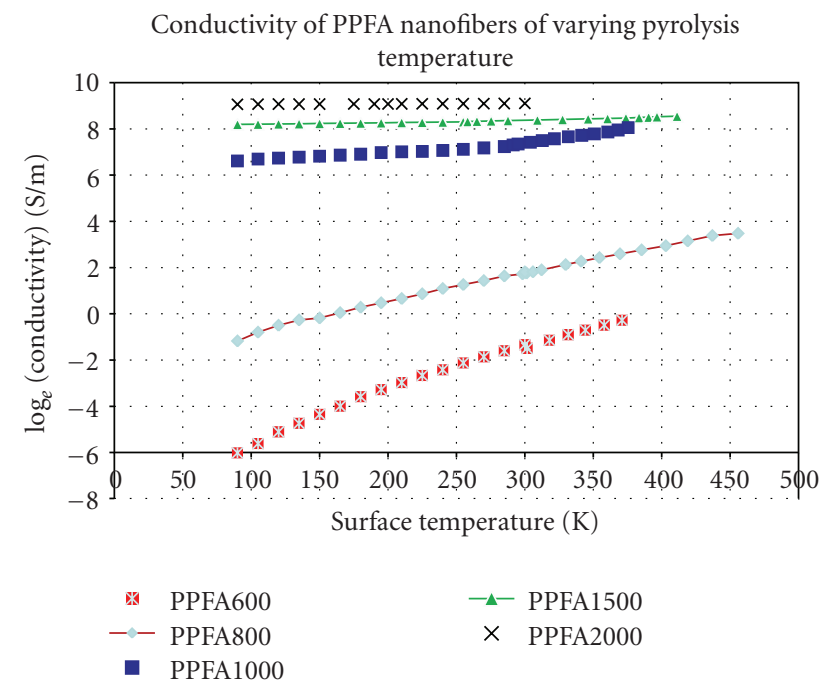

FIGURE 5: Electrical conductivity versus temperature plots for different heat treated specimens.

In Figure 5, the slope of the conductivity versus $T$ curve is highest ( 6 order of magnitude change in resistivity compared to the 3 orders change observed in bulk form [25]) for the PPFA600 sample, and decreases as heat treatment temperature increases. This is seen in both the high and low temperature regions of the data and can be explained by the relative level of disorder present in the material. In glassy semiconductors, defects such as 5- and 7-membered rings cause localized states in the band gap region [41]. The greater the number of localized states, the more the conductivity is dependent on ambient temperature. This is a result of immobile charge carriers, and the activation energy required for excitation of a charge carrier is larger. The increase in ambient temperature therefore allows more charge carriers to cross the mobility gap of the material. With an increase in heat treatment temperature, the system becomes more ordered, and weak, strained bonds are allowed to relax to a more stable $\mathrm{sp}^{2}$ graphitic state. This increase in systemic order means a decrease in the amount of localized states present, and thus more delocalized charge carriers, gradually approaching metallic conduction behavior in which conductivity would not be dependent on ambient temperature. In this situation, an increase in temperature does not provide as significant a change in the amount of electrons that are able to cross. However, the annealing effects decay quickly with the heat treatment temperature. Considering the large scatter in the electrical conductivity data originating from a wide variety of synthesis processes, the nanowire conductivities in this study are found to be marginally higher but in the same order.

To determine the dominant conduction modes in the various temperature regimes, particularly to identify the metal to insulator transition temperature, we examine Figure 6, where individual conductivity versus temperature curves for each heat treatment temperature. From these curves, we can see that for heat treatment temperatures below $800^{\circ} \mathrm{C}$ the conductivity approaches zero as the ambient temperature approaches zero. This is indicative of a hopping mechanism, however, this happens at about $1000^{\circ} \mathrm{C}$ in bulk glassy carbon.

Next, we analyze the data in the lower than room temperature region to establish the parameters for a hoppingbased conduction model (1). A plot of $\ln (\sigma)$ versus $T^{-1 / 2}$ is shown in Figure 7. At higher than the room temperature, all the specimens followed a thermally activated transport mechanism. This is shown in Figure 8, where $\ln (\sigma)$ versus $T^{-1}$ is plotted. The slope of the conductivity versus surface temperature plots is indicative of the activation energy gap, which can be calculated using (3). The slope of this curve is equivalent to $\varepsilon_{g} / k_{B}$, allowing us to determine the mobility gap for the different nanowires. The activation parameters obtained are summarized in Table 1. It is interesting to note that the conductivity data for specimens heat treated at $800^{\circ} \mathrm{C}$ and below do not fit well with the thermally activated conduction mechanism, even at higher than room 


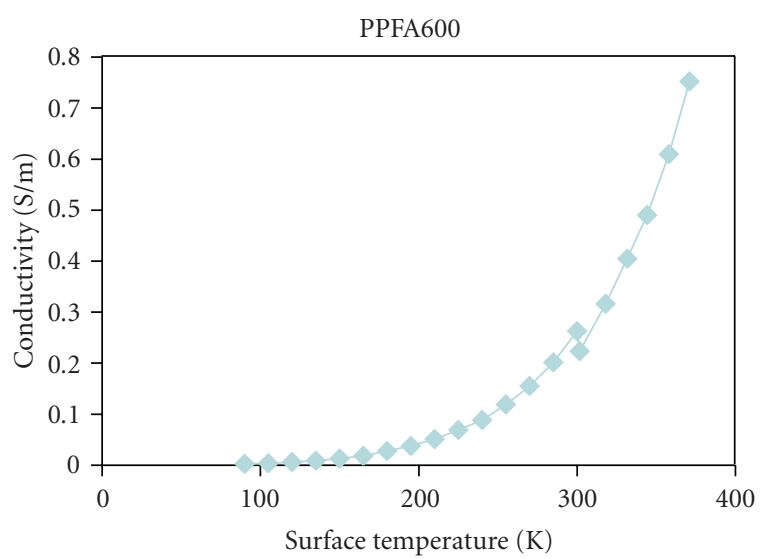

(a)

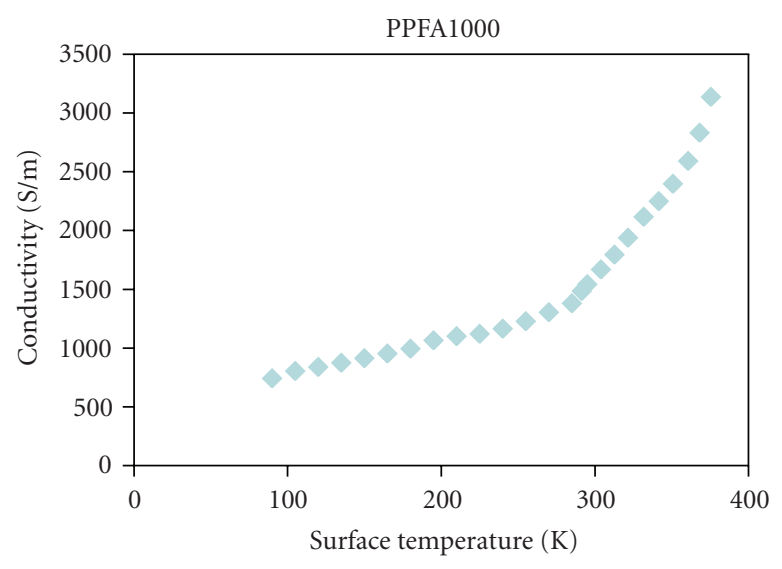

(c)

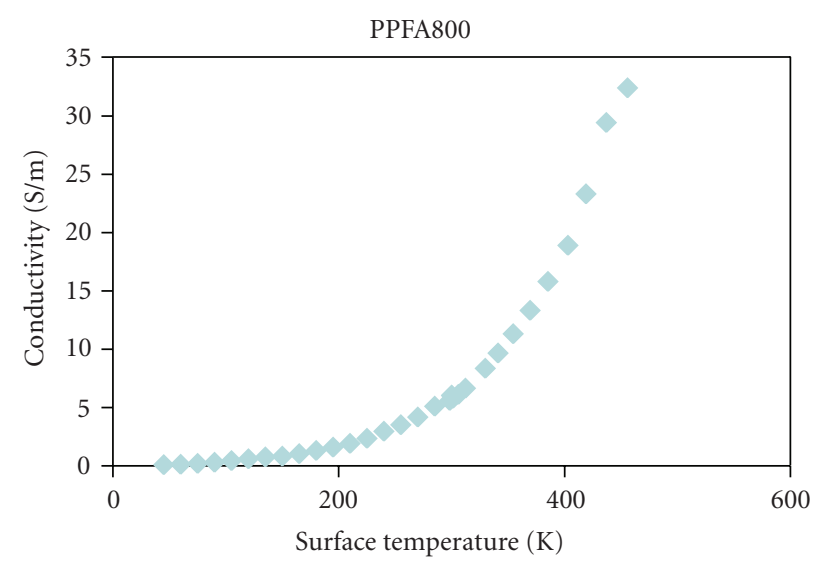

(b)

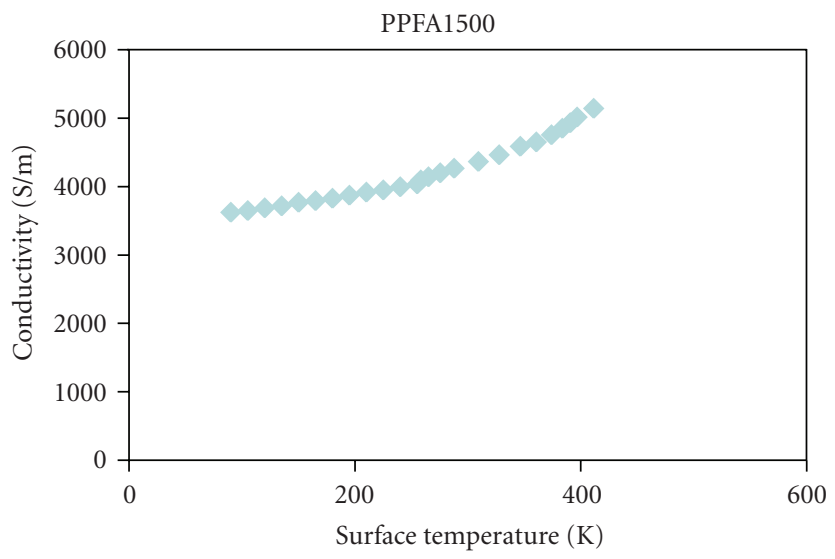

(d)

FIGURE 6: Progressive changes in the trend of conductivity with surface temperature as pyrolysis temperature is increased.

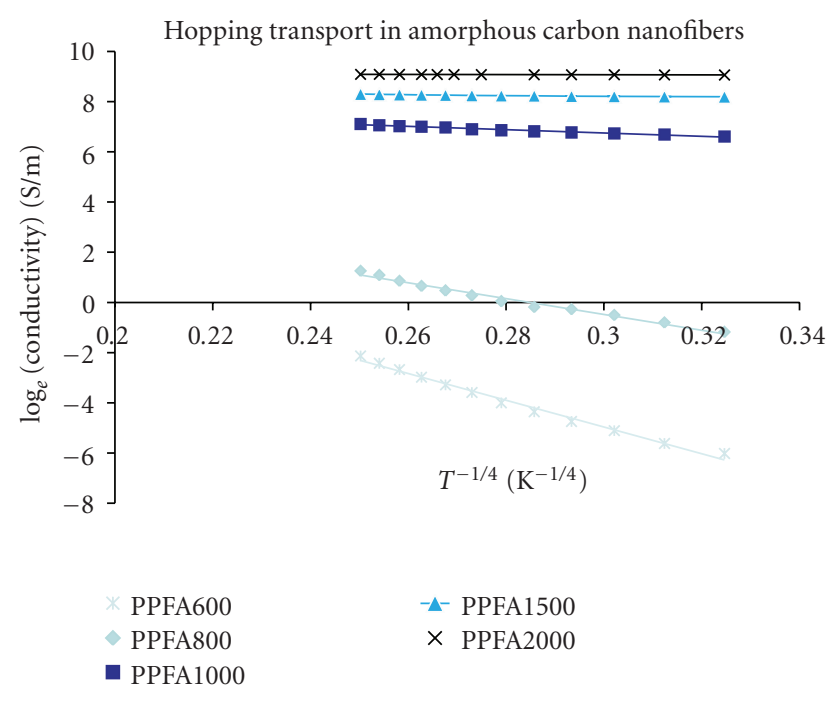

FIGURE 7: Fitting of the low temperature $(<250 \mathrm{~K})$ conductivity data to the hopping transport model.

temperatures. This observation is consistent with the pattern identified in Figure 6.

\section{Discussion}

TEM and Raman microscopy and DC conductivity measurements were performed on individual glassy carbon nanowires with average diameter of $150 \mathrm{~nm}$ in an attempt to associate the heat treatment temperature and the corresponding nanowire microstructure with the electrical properties of the material. The TEM images show a significant increase in graphitic, ribbon-like structures as the heat treatment temperature was raised from $600^{\circ} \mathrm{C}$ to $2000^{\circ} \mathrm{C}$. The Raman spectra also qualitatively indicated similar increase in systemic order. DC conductivity measurements agree to this trend quantitatively, with 6 orders of magnitude increase in conductivity as the ambient temperature increase from $90 \mathrm{~K}$ to $450 \mathrm{~K}$. The slope of the conductivity versus temperature curve is highest (in magnitude) for the lowest heat treatment temperature, and decreases as heat treatment temperature increases. This is seen in both the high and low temperature regions of the data and can be explained by the relative level of disorder present in the material, which determines the amount of localized states, and therefore the temperature dependence of the material. As surface temperature is increased, more localized electrons have the energy to bridge the gap. With an increase in heat treatment 
TABle 1: Parameters from the low temperature DC conductivity data fitted to both a hopping-based energy transport mechanism and a thermally activated conduction mechanism.

\begin{tabular}{lcccc}
\hline Sample & Slope for hopping conduction & $R^{2}$ for hopping conduction model & Mobility gap, $\varepsilon_{g}(\mathrm{eV})$ & $\begin{array}{c}R^{2} \text { for thermally activated } \\
\text { conduction model }\end{array}$ \\
\hline PPFA600 & 92.607 & 0.98978 & 0.0033 & 0.98117 \\
PPFA800 & 54.959 & 0.98181 & 0.0152 & 0.97261 \\
PPFA1000 & 11.451 & 0.98183 & 0.0779 & 0.97225 \\
PPFA1500 & 2.5482 & 0.95712 & 0.1309 & 0.9413 \\
PPFA2000 & 0.5533 & 0.94543 & 0.1664 & 0.95255 \\
\hline
\end{tabular}

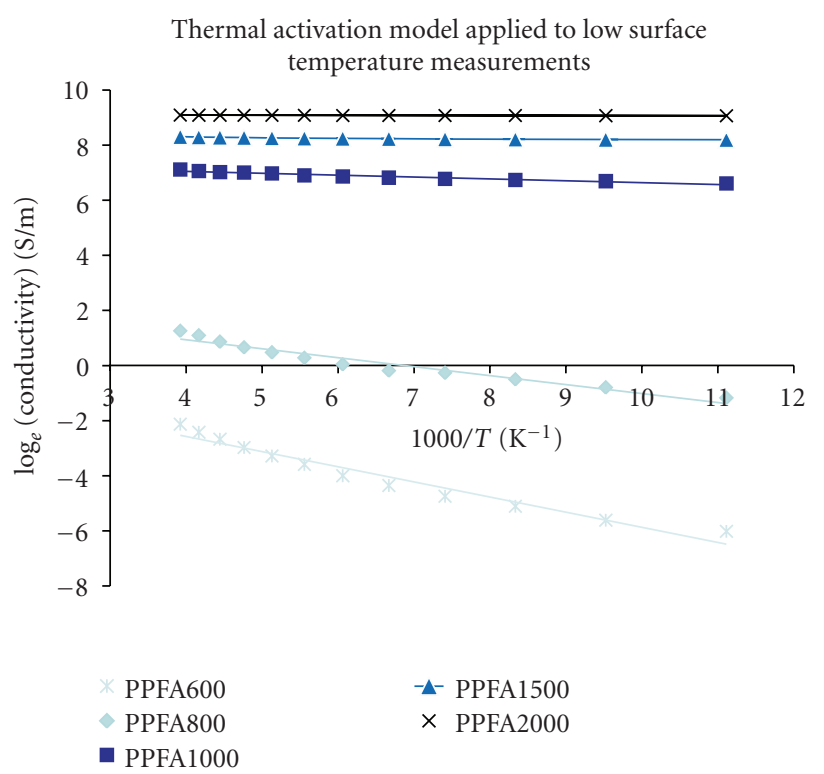

FIGURE 8: Fitting of the conductivity data for room temperature and above to a thermally activated transport model.

temperature, the system becomes more ordered, decreasing the amount of localized states present and allowing for more mobile charge carriers. With this increased electron mobility, an increase in temperature does not provide as significant a change in the amount of electrons that are able to cross, and the conductivity of the sample does not increase as rapidly with increasing surface temperature.

We conclude that it is the overall delocalized charge carrier density which determines the [41] conductivity of the system. This result can also be connected to the morphology of the nanowires. When the system is highly disordered, there are significantly fewer available charge carriers, as they are involved in the defects and strained bonds in the structure. At higher heat treatment temperatures, much of this disorder is removed from the system, which improves the conductivity of the nanowires. This is dominated by the chemical processes during heat treatment such as increases in aromatic $\mathrm{sp}^{2}$ ring formation, growth of curved graphitic crystallite domains, increase in percolation networks, and the annealing of graphitic defects.

\section{Acknowledgments}

M.A. Haque gratefully acknowledges the support from the Center for Nanoscale Mechatronics \& Manufacturing of the Korea Institute of Machinery \& Materials and the National Science Foundation, USA (ECS no. 0545683).

\section{References}

[1] S. R. P. Silva and J. D. Carey, "Enhancing the electrical conduction in amorphous carbon and prospects for device applications," Diamond and Related Materials, vol. 12, no. 2, pp. 151-158, 2003.

[2] J. Robertson, "Preparation and properties of amorphous carbon,” Journal of Non-Crystalline Solids, vol. 137-138, no. 2, pp. 825-830, 1991.

[3] S. R. P. Silva, Ed., Properties of Amorphous Carbon, vol. 29 of EMIS Data Reviews Series, Institution of Engineering and Technology, London, UK, 2003.

[4] G. M. Jenkins, K. Kawamura, and L.L. Ban, "Formation and structure of polymeric carbons," Proceedings of the Royal Society of London. Series A, Mathematical and Physical Sciences, vol. 327, no. 1571, pp. 501-517, 1972.

[5] C. L. Burket, R. Rajagopalan, and H. C. Foley, "Overcoming the barrier to graphitization in a polymer-derived nanoporous carbon," Carbon, vol. 46, no. 3, pp. 501-510, 2008.

[6] D. G. McCulloch, J. L. Peng, D. R. McKenzie, S. P. Lau, D. Sheeja, and B. K. Tay, "Mechanisms for the behavior of carbon films during annealing," Physical Review B, vol. 70, no. 8, Article ID 085406, 8 pages, 2004.

[7] D. F. Baker and R. H. Bragg, "The electrical conductivity and Hall effect of glassy carbon," Journal of Non-Crystalline Solids, vol. 58, no. 1, pp. 57-69, 1983.

[8] E. P. O'Reilly, "The electronic structure of amorphous carbon," Journal of Non-Crystalline Solids, vol. 97-98, no. 2, pp. 10951102, 1987.

[9] R. R. Saxena and R. H. Bragg, "Electrical conduction in glassy carbon," Journal of Non-Crystalline Solids, vol. 28, no. 1, pp. 45-60, 1978.

[10] C. L. Burket, R. Rajagopalan, A. P. Marencic, K. Dronvajjala, and H. C. Foley, "Genesis of porosity in polyfurfuryl alcohol derived nanoporous carbon," Carbon, vol. 44, no. 14, pp. 2957-2963, 2006.

[11] D. Sun, L. Zhu, and G. Zhu, "Glassy carbon ceramic composite electrodes," Analytica Chimica Acta, vol. 564, no. 2, pp. 243247, 2006.

[12] S. Shahrokhian, M. Ghalkhani, M. Adeli, and M. K. Amini, "Multi-walled carbon nanotubes with immobilised cobalt 
nanoparticle for modification of glassy carbon electrode: application to sensitive voltammetric determination of thioridazine," Biosensors and Bioelectronics, vol. 24, no. 11, pp. 32353241, 2009.

[13] Q. Ngo, T. Yamada, M. Suzuki et al., "Structural and electrical characterization of carbon nanofibers for interconnect via applications," IEEE Transactions on Nanotechnology, vol. 6, no. 6, pp. 688-695, 2007.

[14] R. Martinez-Duarte et al., "Perspectives of Micro and Nanofabrication of Carbon for Electrochemical and Microfluidic Applications," in Microfluidics and Microfabrication, S. Chakraborty, Ed., pp. 181-263, Springer, New York, NY, USA, 2010.

[15] P. Yu, Y. Lin, L. Xiang, L. Su, J. Zhang, and L. Mao, "Molecular films of water-miscible ionic liquids formed on glassy carbon electrodes: characterization and electrochemical applications," Langmuir, vol. 21, no. 20, pp. 9000-9006, 2005.

[16] C. Wang, L. Taherabadi, G. Jia, M. Madou, Y. Yeh, and B. Dunn, "C-MEMS for the manufacture of 3D microbatteries," Electrochemical and Solid-State Letters, vol. 7, no. 11, pp. A435A438, 2004.

[17] A. Singh, J. Jayaram, M. Madou, and S. Akbar, "Pyrolysis of negative photoresists to fabricate carbon structures for microelectromechanical systems and electrochemical applications," Journal of the Electrochemical Society, vol. 149, no. 3, pp. E78E83, 2002.

[18] C. Wang and M. Madou, "From MEMS to NEMS with carbon," Biosensors and Bioelectronics, vol. 20, no. 10, pp. 2181-2187, 2005.

[19] R. Du, S. Ssenyange, M. Aktary, and M. T. McDermott, "Fabrication and characterization of graphitic carbon nanostructures with controllable size, shape, and position," Small, vol. 5, no. 10, pp. 1162-1168, 2009.

[20] C. S. Sharma, R. Vasita, D. K. Upadhyay, A. Sharma, D. S. Katti, and R. Venkataraghavan, "Photoresist derived electrospun carbon nanofibers with tunable morphology and surface properties," Industrial and Engineering Chemistry Research, vol. 49, no. 6, pp. 2731-2739, 2010.

[21] S. E. Rodil, R. Olivares, H. Arzate, and S. Muhl, "Biocompatibility, cytotoxicity and bioactivity of amorphous carbon films," Topics in Applied Physics, vol. 100, pp. 55-75, 2006.

[22] V.-M. Tiainen, "Amorphous carbon as a bio-mechanical coating-mechanical properties and biological applications," Diamond and Related Materials, vol. 10, no. 2, pp. 153-160, 2001.

[23] R. McCreery, J. Dieringer, A. O. Solak et al., "Molecular rectification and conductance switching in carbon-based molecular junctions by structural rearrangement accompanying electron injection," Journal of the American Chemical Society, vol. 125, no. 35, pp. 10748-10758, 2003.

[24] F. Anariba, S. H. DuVall, and R. L. McCreery, "Mono-and multilayer formation by diazonium reduction on carbon surfaces monitored with atomic force microscopy "scratching", Analytical Chemistry, vol. 75, no. 15, pp. 3837-3844, 2003.

[25] W. Bücker, "Preparation and DC conductivity of an amorphous organic semiconducting system," Journal of NonCrystalline Solids, vol. 12, no. 1, pp. 115-128, 1973.

[26] L. Soukup, I. Gregora, L. Jastrabik, and A. Koňáková, "Raman spectra and electrical conductivity of glassy carbon," Materials Science and Engineering B, vol. 11, no. 1-4, pp. 355-357, 1992.

[27] B. Y. Park, L. Taherabadi, C. Wang, J. Zoval, and M. J. Madou, "Electrieal properties and shrinkage of carbonized photoresist films and the implications for carbon microelectromechanical systems devices in conductive media," Journal of the Electrochemical Society, vol. 152, no. 12, pp. J136-J143, 2005.

[28] B. A. Samuel, R. Rajagopalan, H. C. Foley, and M. A. Haque, "Temperature effects on electrical transport in semiconducting nanoporous carbon nanowires," Nanotechnology, vol. 19, no. 27, Article ID 275702, 2008.

[29] R. Gonzalez, R. Martinez, and P. Ortiz, "Polymerization of furfuryl alcohol with trifluoroacetic acid: the influence of experimental conditions," Macromolecular Chemistry, vol. 193, no. 1, pp. 1-9, 1992.

[30] E. Fitzer and W. Schäfer, "The effect of crosslinking on the formation of glasslike carbons from thermosetting resins," Carbon, vol. 8, no. 3, pp. 353-364, 1970.

[31] Z. Wang, Z. Lu, Y. Huang, R. Xue, X. Huang, and L. Chen, "Characterizations of crystalline structure and electrical properties of pyrolyzed polyfurfuryl alcohol," Journal of Applied Physics, vol. 82, no. 11, pp. 5705-5710, 1997.

[32] K. Dasgupta and D. Sathiyamoorthy, "Disordered carbonits preparation, structure, and characterisation," Materials Science and Technology, vol. 19, no. 8, pp. 995-1002, 2003.

[33] K. Takai, M. Oga, H. Sato et al., "Structure and electronic properties of a nongraphitic disordered carbon system and its heat-treatment effects," Physical Review B, vol. 67, no. 21, Article ID 214202, 11 pages, 2003.

[34] K. Kuriyama and M. S. Dresselhaus, "Metal-insulator transition in highly disordered carbon fibers," Journal of Materials Research, vol. 7, no. 4, pp. 940-945, 1992.

[35] J. P. Sullivan, T. A. Friedmann, and A. G. Baca, "Stress relaxation and thermal evolution of film properties in amorphous carbon," Journal of Electronic Materials, vol. 26, no. 9, pp. 1021-1029, 1997.

[36] A. Tibrewala, E. Peiner, R. Bandorf, S. Biehl, and H. Lüthje, "Transport and optical properties of amorphous carbon and hydrogenated amorphous carbon films," Applied Surface Science, vol. 252, no. 15, pp. 5387-5390, 2006.

[37] V. Ambegaokar, B. I. Halperin, and J. S. Langer, "Hopping conductivity in disordered systems," Physical Review B, vol. 4, no. 8, pp. 2612-2620, 1971.

[38] B. Y. Park, L. Taherabadi, C. Wang, J. Zoval, and M. J. Madou, "Electrieal properties and shrinkage of carbonized photoresist films and the implications for carbon microelectromechanical systems devices in conductive media," Journal of the Electrochemical Society, vol. 152, no. 12, pp. J136-J143, 2005.

[39] B. A. Samuel, R. Rajagopalan, H. C. Foley, and M. A. Haque, "Temperature effects on electrical transport in semiconducting nanoporous carbon nanowires," Nanotechnology, vol. 19, no. 27, Article ID 275702, 2008.

[40] A. C. Ferrari and J. Robertson, "Interpretation of Raman spectra of disordered and amorphous carbon," Physical Review B, vol. 61, no. 20, pp. 14095-14107, 2000.

[41] A. Takshi, M. Mohammadi, and J. D. Madden, "Study the effect of distribution of density of states on the depletion width of organic Schottky contacts," Solid-State Electronics, vol. 52, no. 11, pp. 1717-1721, 2008. 

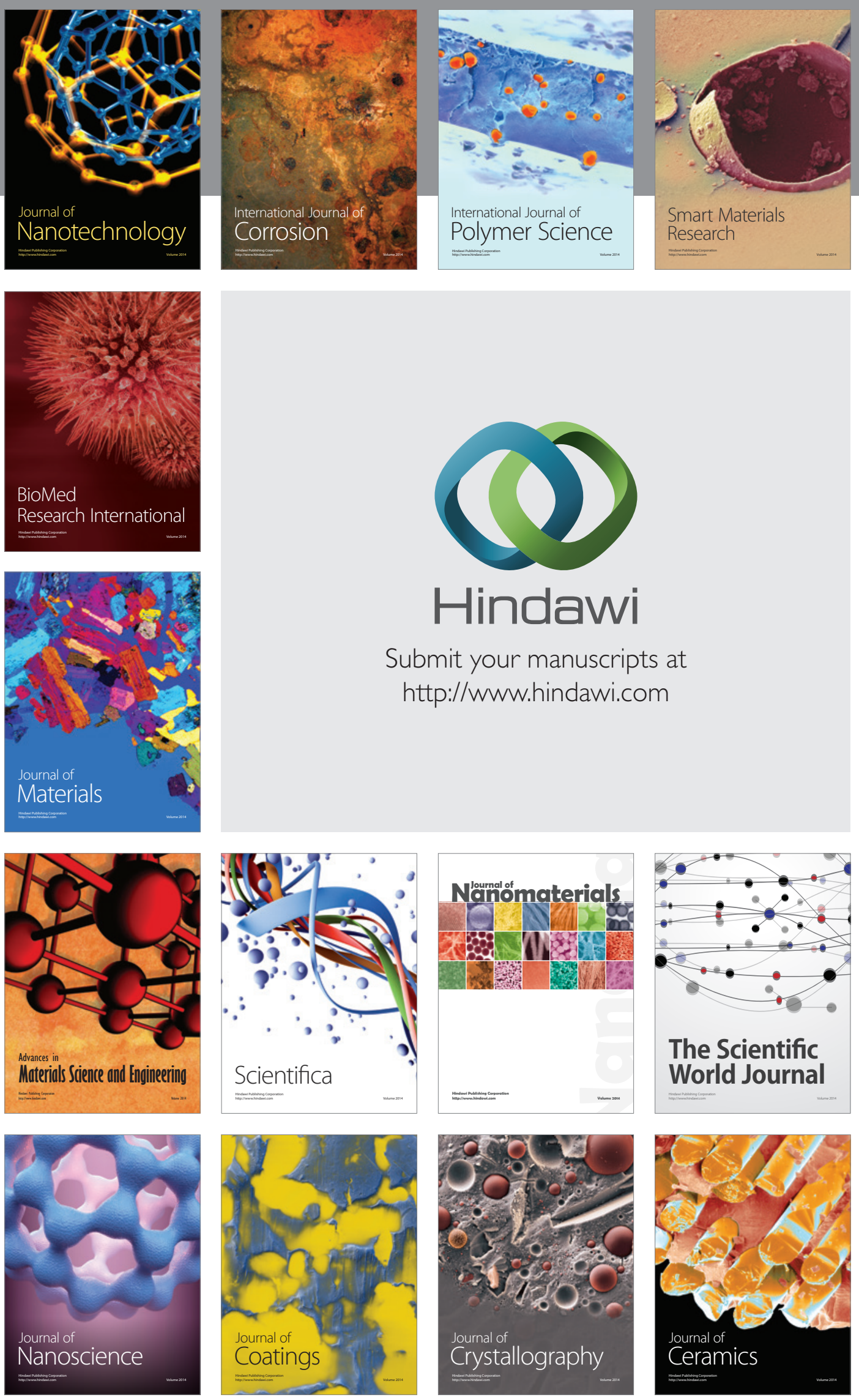

The Scientific World Journal

Submit your manuscripts at

http://www.hindawi.com

\section{World Journal}

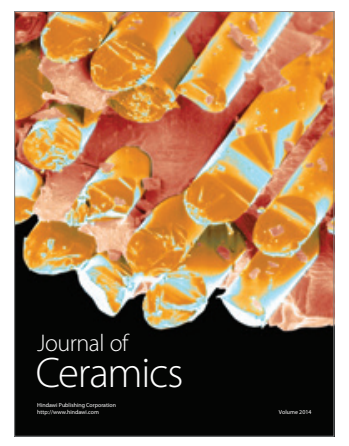

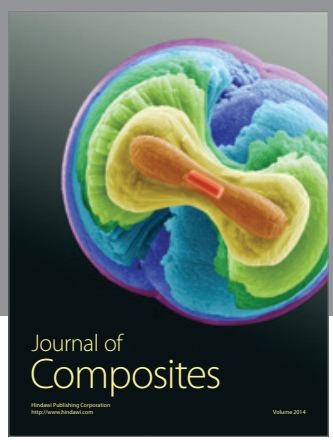
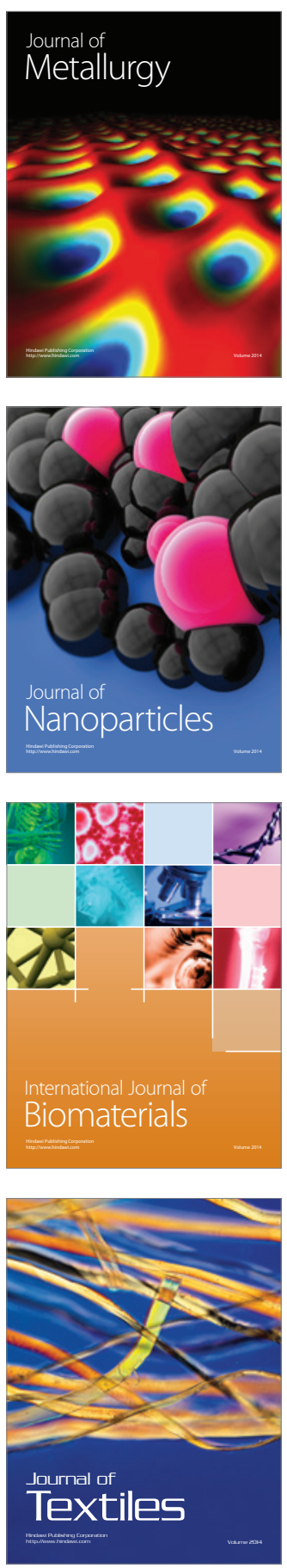